\title{
Curing Reaction and Dielectric Properties of Rigid and Elastic Liquid Crystal Epoxy Networks Modified with Nanofillers
}

\author{
Magdalena Włodarska (iD) \\ Institute of Physics, Lodz University of Technology, Wólczańska 219, 90-924 Łódź, Poland \\ Correspondence should be addressed to Magdalena Włodarska; magdaw@p.lodz.pl
}

Received 30 April 2018; Revised 27 June 2018; Accepted 5 July 2018; Published 1 August 2018

Academic Editor: Ulrich Maschke

Copyright (c) 2018 Magdalena Włodarska. This is an open access article distributed under the Creative Commons Attribution License, which permits unrestricted use, distribution, and reproduction in any medium, provided the original work is properly cited.

\begin{abstract}
The aim of the paper is to compare the progress of the curing reaction and the dielectric properties of liquid crystalline epoxy networks of different elasticity and to investigate how modification with nanofillers influences their properties. The study focuses on a liquid crystalline epoxy monomer with four aromatic rings in the mesogen, cross-linked with $4,4^{\prime}$-diaminodiphenylmethane (DDM) and pimelic acid (PA) to produce rigid and elastic polymer networks. The obtained results are compared to networks modified with nanofillers (diphenyl aluminum phosphate nanorods). The curing process is monitored in situ with broadband dielectric spectroscopy, which is a good tool to view the progress of the reaction. Two stages with different reaction dynamics are observed in the studied cases. Dielectric properties of the products cured with two chosen agents show significant differences in the obtained parameters (activation energy, glass transition, and fragility index) as well as in the dynamics of the $\alpha$ and $\beta$ relaxations. Although the curing agent is the main factor determining the properties of the product, the nanofiller additive also modifies the values of specific parameters that characterize both the process of the reaction and the properties of the final composites. Particularly, adding the nanofiller raises the glass transition temperature in both the cases. The obtained results are in good agreement with the earlier calorimetric study.
\end{abstract}

\section{Introduction}

Although epoxy resins have already found a wide range of industrial applications, there is a continuing interest in these materials, aiming at the development of advanced composites with specific thermal, electrical, and mechanical properties [1-14]. For example, epoxy materials have high thermal, mechanical, and electrical stability, which is highly demanded by the industry [1-4]. Another big advantage of epoxy resins is the prospect of modifying their selected properties by using organic and inorganic materials or nanoparticles as fillers and building advanced composites [4, 7-11]. A separate group of such materials are epoxy resins based on liquid crystalline mesogens, which are used to produce liquid crystalline epoxy networks (LCEN) $[1-3,5,6]$. Due to the structure of LCEN, their properties and curing conditions are different and more complex than those of traditional resins $[6,12-14]$. Both the curing process and the properties of the cured products depend on various factors such as the curing agent, curing conditions, or addition of nanofillers, as well as the properties of the pure epoxy resin such as the type of mesophase $[5,6,12]$.

Many techniques can be used to describe the properties of the final resin; one of them is the dielectric relaxation spectroscopy (DRS). This method is very sensitive to changes in the molecular properties of the material. In consequence, it is frequently used for observation of phase transitions or monitoring of the reaction progress. The dielectric response is usually represented in terms of the complex electric permittivity $\varepsilon^{*}$ as a function of frequency and temperature (or frequency and time, in the in situ experiments). The principle of dielectric spectroscopy is based on measuring changes in the dielectric properties of the sample in response to the applied small, alternating electric field. As a result, several parameters can be observed-such as the electric conductivity, the dielectric constant connected with location of diverse dipole moments and their cooperation in the material, and some relaxation processes (with characteristic times) 
which result from delayed reaction on sample polarization. These parameters, describing the electrical behavior of the sample, can also be linked with some mechanical properties. For example, in polymers, at least two relaxation processes (denoted with Greek letters $\alpha$ and $\beta$ ) are most frequently observed [15-21]. The structural $\alpha$-process usually corresponds to the glass transition. It is thereby related to hindering of the motion of polymer chain segments, caused by approaching the vitrification temperature $T_{\mathrm{g}}$. The $\alpha$-process is typically the slowest one in polymers (appearing at the lowest frequencies/the highest temperatures). The $\beta$-process occurs at higher frequencies/lower temperatures and is believed to involve rotational motions of polar group fragments containing single bonds within the molecule [17-19]. These motions can be treated as almost free rotations of dipolar groups. The $\alpha$ - and $\beta$-processes exhibit different temperature dependencies. The relaxation time of the molecular $\beta$-process can usually be described with the exponential Arrhenius formula, but deviations from the exponential dependency may be observed near the vitrification temperature, where the structural $(\alpha)$ process appears. For the description of dynamic properties of glass, such as the structural relaxation time, an empirical formula was proposed by Vogel, Fulcher, and Tammann (VFT) [17-21]. Although the complex permittivity $\varepsilon^{*}$ is most commonly used to discuss the results, another representation was proposed for polymers with high ionic conductivity: the electric modulus [22]. It is defined as the inverse of the complex permittivity [22-24]:

$$
M^{*}=\frac{1}{\varepsilon^{*}} .
$$

This quantity was introduced by analogy with the modulus observed in mechanical studies. The modulus representation is particularly convenient when no relaxation processes occur at a given temperature and ionic conductivity is high. Then, the imaginary component of the electric modulus $\left(M^{\prime \prime}\right)$ displays a characteristic peak which is directly related to ionic conductivity [22]:

$$
\tau_{\sigma}=\frac{\varepsilon_{0} \varepsilon_{s}}{\sigma_{0}}=\frac{\varepsilon_{0}}{M_{S} \cdot \sigma_{0}} .
$$

Here, $\tau_{\sigma}$ can be interpreted as the conductivity relaxation time (the inverse of the frequency of the maximum of $M^{\prime \prime}$ ), $\varepsilon_{0}$ is the electric permittivity of free space $\left(8.854^{*} 10^{-14} \mathrm{~F} / \mathrm{cm}\right)$, and $\varepsilon_{s}$ is the frequency-independent relative permittivity which is linked with the real part of the electric modulus:

$$
\lim _{\omega \tau_{\sigma} \gg 1} M^{\prime}=M_{s}=\frac{1}{\varepsilon_{s}} .
$$

On the other side, the real part of the modulus approaches zero at low frequencies:

$$
\lim _{\omega \tau_{\sigma} \ll 1} M^{\prime}=0 .
$$

In this work, the modulus representation is used to monitor the reaction. This approach has already been successfully applied to in situ observation of curing reactions. Early papers, in which dielectric spectroscopy was employed to study curing of commercially available materials, demonstrated its usefulness $[25,26]$. Nowadays, the technique continues to be developed with the aim of extracting more information, from the dielectric response in the course of the curing reaction to its connection with characteristic parameters of the reaction, such as the reaction rate.

Dielectric response of liquid crystalline epoxy compounds is quite complex due to the presence of polar, rigid mesogenic units. It is known that dielectric spectra of a liquid crystal consist of different relaxation processes which depend on the direction of the electric field with respect to the orientation of the mesogens. In unordered samples of lowmolecular mass liquid crystals, one (broadened) or two relaxation modes are usually observed by virtue of overlapping of all the existing relaxation types [20, 27-30]. Hence, in cured liquid crystalline epoxies, various relaxation processes connected with different relaxation modes may appear; this has a potential for providing more information about the reaction and the molecular behavior in the final material. Dielectric studies on materials such as liquid crystalline epoxy networks (LCEN) not only facilitate a better understanding of the processes occurring in the material but also enable correlating distinct features of the dielectric response with specific properties of the sample. Additionally, building composites based on LCEN allows for modification of specific properties of the obtained network while keeping other properties unchanged.

In this work, one liquid crystalline epoxy monomer with a long mesogenic group (containing four aromatic rings) was chosen for the investigation. The synthetic route and the best curing conditions for this material were established in a previous work [31]. 4,4'-Diaminodiphenylmethane (DDM) and pimelic acid (PA) were selected as curing agents to obtain rigid and elastic networks. The size of the mesogen causes the phase transitions to occur at relatively high temperatures, which is usually a disadvantage; but it is beneficial for the curing process-which also takes place at elevated temperatures-because it facilitates carrying out of the process in the liquid crystalline state. The results were supplemented by a comparison of the studied materials with the same polymer matrices containing a nanofiller. Dielectric spectroscopy was applied to study both the curing process and the properties of the cured products. The aim of the author was to show differences in the dielectric responses of rigid and more elastic LCEN, by using two different curing agents. An additional modification of the LCEN with nanofillers resulted in increased rigidity of both the products. However, these changes had a different character than those related to the selection of the curing agent.

\section{Materials}

The molecular structures of the monomer and the curing agents are shown in Figure 1. All the reagents and curing agents $\left(4,4^{\prime}\right.$-diaminodiphenylmethane (DDM) and pimelic acid (PA)) were commercial (Sigma-Aldrich, Fluka, POCH, Gliwice) and were used without further purification. 


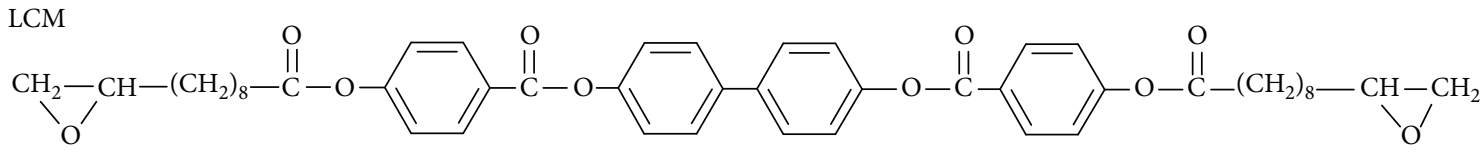

$\operatorname{DDM}\left(4,4^{\prime}\right.$-diaminodiphenylmethane)

PA (pimelic acid)

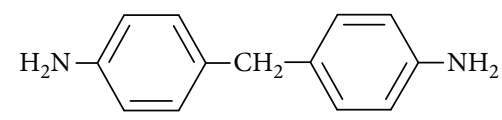

$\stackrel{\mathrm{O}}{\mathrm{HO}}-\stackrel{\mathrm{C}}{\mathrm{C}}-\left(\mathrm{CH}_{2}\right)_{5}-\stackrel{\mathrm{O}}{\mathrm{C}}-\mathrm{OH}$

FIGURE 1: Molecular structure of the epoxy monomer and the curing agents.

TABLE 1: Temperatures of phase transitions in the pure liquid crystal epoxy monomer.

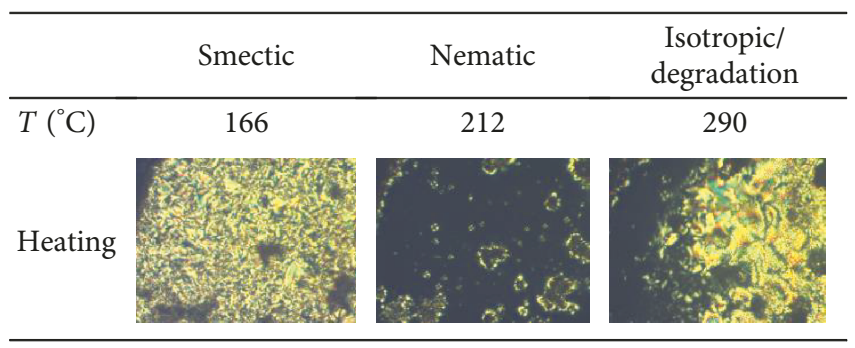

Temperatures of phase transitions found in the previous study were confirmed by optical data and are presented in Table 1 . The curing conditions chosen for the dielectric study were the same for all the materials-isothermal curing at $170^{\circ} \mathrm{C}$ for 2.5 hours followed by postcuring at $210^{\circ} \mathrm{C}$ for 1.5 hours-and comparable with those for the previous study [31]. The earlier DSC study of the investigated mixtures also enabled determination of the optimal curing conditions. DSC analysis of the cured products did not reveal any phase transitions (except vitrification) [31]. Both compositions were subsequently cured with the addition of a nanofiller, in the same conditions. Diphenyl aluminum phosphate nanorods (NR) having $100-200 \mathrm{~nm}$ in diameter and $1-5 \mu \mathrm{m}$ in length were used as the filler. The details of the synthesis of the nanorods and their properties were described elsewhere [32, 33].

\section{Experimental}

The dielectric data during isothermal curing was recorded in the frequency range from $10^{-2}$ to $10^{5} \mathrm{~Hz}$ using Solartron 1260 Impedance Analyzer with Chelsea Dielectric Interface. The mixtures were placed between two parallel metal electrodes with diameters of 5 and $10 \mathrm{~mm}$, separated by $50 \mu \mathrm{m}$ silica spacers. The temperature of the samples was stabilized using Unipan 620 temperature controller with the accuracy of $0.05 \mathrm{~K}$. The duration of a single-frequency sweep at the curing temperature of $170^{\circ} \mathrm{C}$ was about 3 minutes. The next series of measurements was started after 4 minutes (in most of the cases), yielding a 3D plot with a 4-minute separation between subsequent data points at a given frequency.
Measurements in a wide range of temperatures for all the cured products were performed with an alpha highresolution dielectric analyzer coupled with a Quatro Cryosystem, providing isothermal conditions and temperature stabilization of $0.1 \mathrm{~K}$ (both devices are manufactured by Novocontrol). Data was collected in the cooling route in a wide range of frequency $\left(10^{6}-10^{-1} \mathrm{~Hz}\right)$.

The optical observations were made using a polarizing microscope with crossed polarizers and a BIC-2S $1 / 2^{\prime \prime}$ CMOS digital camera. The material was placed between plain glass plates in a Linkam HFS600E-PB4 hot stage.

\section{Results and Discussion}

4.1. Process of Curing. The electric modulus representation can be used for in situ monitoring of the curing process of epoxy resins. The data collected in the course of curing the investigated monomer with DDM is shown in Figure 2(a).

For comparison, Figure 2(b) shows the process of curing with PA. The shift in the frequency of the electric modulus peak is connected, as mentioned earlier, with the changes in the electric conductivity. In principle, the curing reaction in epoxy materials is biphasic. The first, highly exothermic stage, characterized with rapid changes of the conductivity, lasts between a few minutes and a few tens of minutes. However, the mixture does not attain its final state and properties in that phase yet. In the second stage, slow evolution of certain properties can still be observed, until they finally stabilize after several hours. Sometimes, postcuring treatment at an elevated temperature is required for complete stabilization of the product. Dielectric studies allow for monitoring of the full progress of curing, since both phases of the reaction are accompanied by changes in the dielectric response. A comparison of the two plots shows that the initial, rapid phase ends much sooner in the LCM/DDM mixture (Figure 2(a)) than that in the case when pimelic acid was used as the curing agent (Figure 2(b)). Different reaction kinetics is related to different functionalities of the reagents (DDM is tetrafunctional and PA is difunctional), which results in a higher cross-link density and a more rigid network cured with DDM. An additional explanation of the different reaction kinetics is the presence of steric constraints in the cured product, which may significantly slow down the process: when PA is used, no extra aromatic rings are added to the composition and the reaction can progress rapidly for a longer time. This observation, based on comparing the reaction 


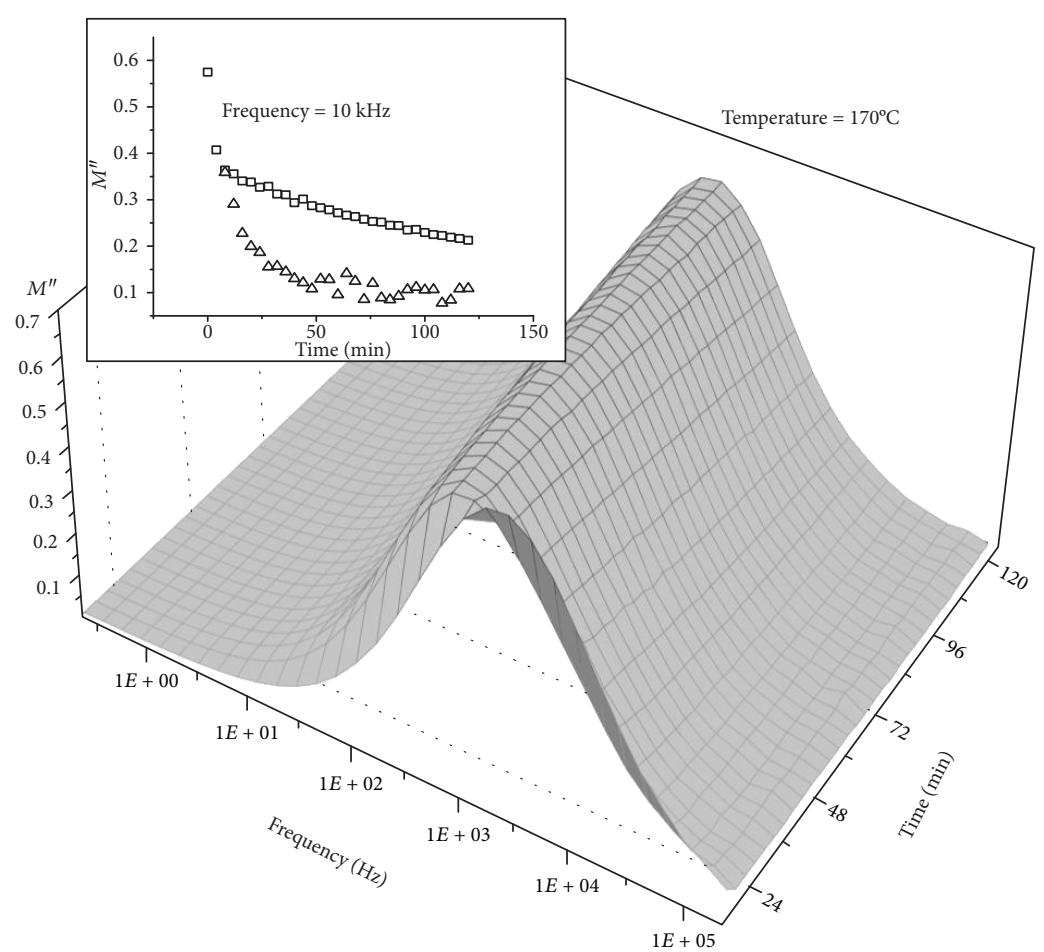

LCM/DDM

LCM/DDM_N

(a)

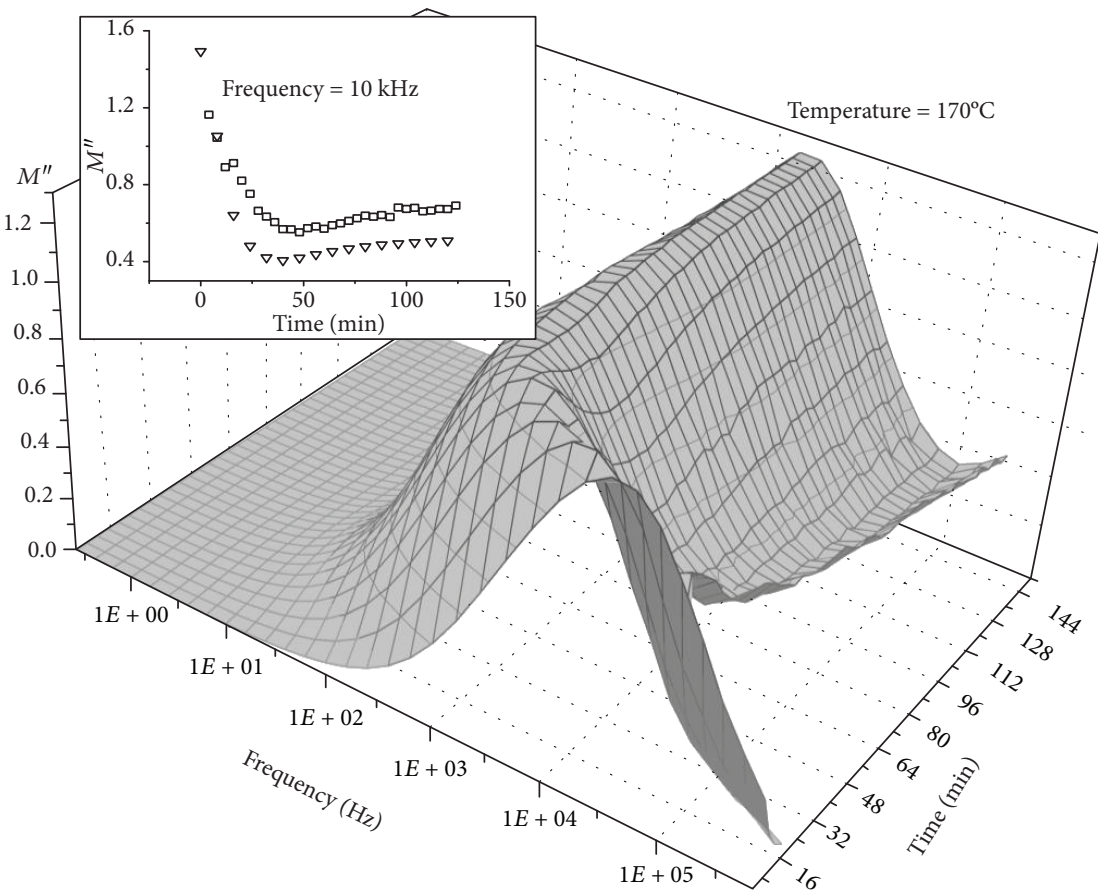

LCM/PA

$\checkmark$ LCM/PA_N

(b)

FIgURE 2: Isothermal curing of the epoxy resin at $T=170^{\circ} \mathrm{C}$, in the electric modulus representation. Imaginary part of the electric modulus plotted versus time in a wide frequency range: (a) curing with the aromatic amine (DDM); (b) curing with pimelic acid (PA). Insets: modulus evolution with time at one specific frequency, for mixtures cured with and without nanofillers. 


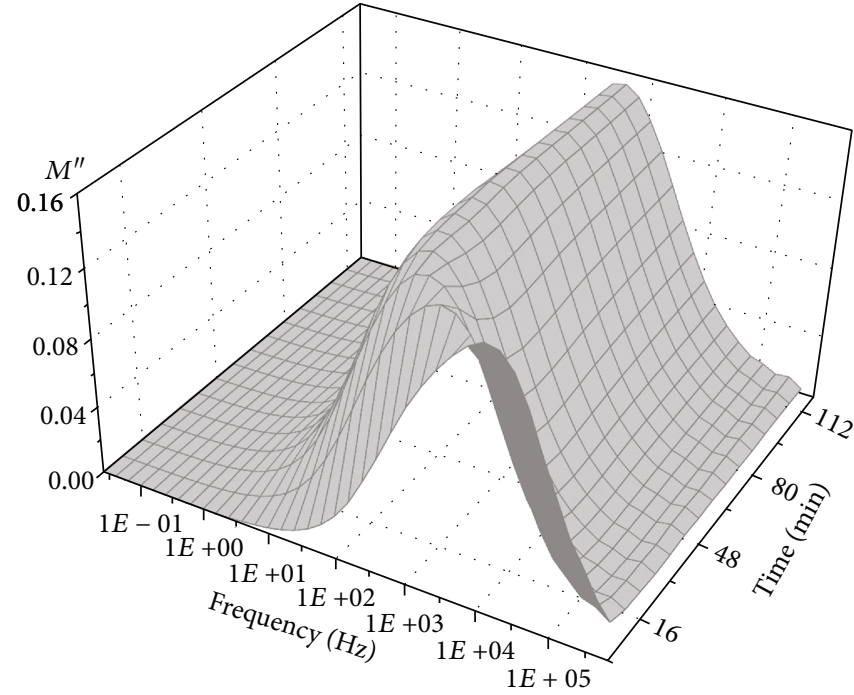

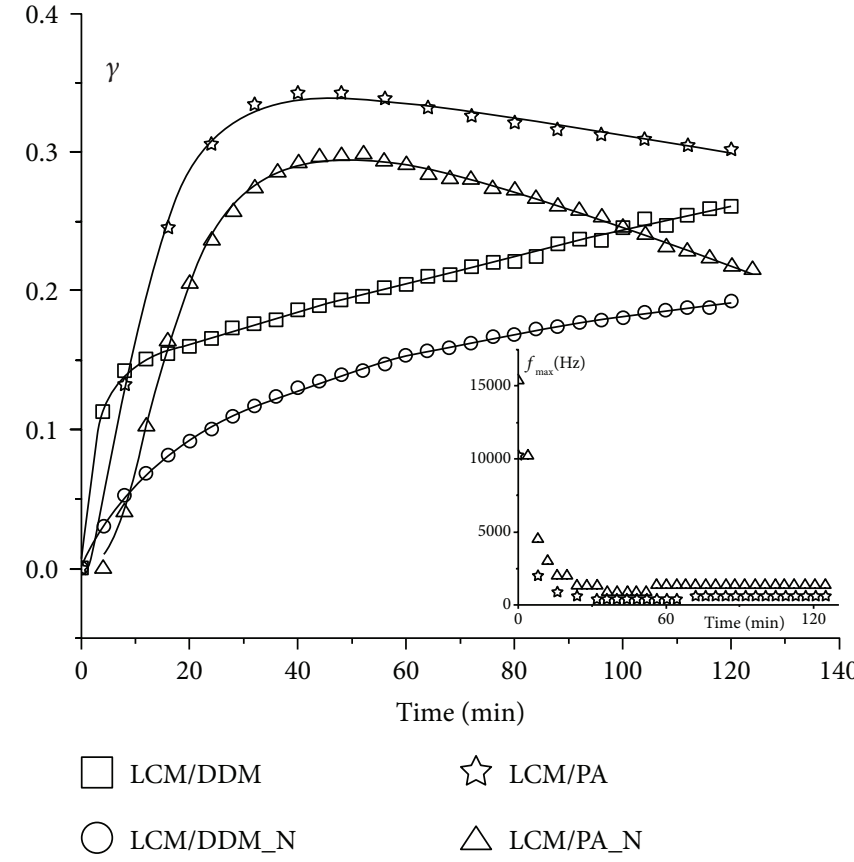

(b)

Figure 3: Curing process of the epoxy resin and its composites: (a) 3D representation (electric modulus versus frequency and time) of curing of the epoxy resin with pimelic acid and nanorods; (b) normalized real part of permittivity $(\gamma)$ versus time for all the investigated mixtures, with solid lines representing the best fit of the chosen kinetic model to the data (inset: estimated frequency of the $M^{\prime \prime}$ peak versus time for composites cured with pimelic acid).

profiles, was confirmed by the study of the reaction products: indeed, more elastic materials were obtained from the mixtures cured with PA.

Introducing the nanofiller does not fundamentally change the kinetics of the curing reaction in either case, which depends mainly on the properties of the curing agent. The reaction profiles are almost identical for the composites cured with DDM, with and without the nanofiller. Small differences are visible between the composites cured with PA-as shown for comparison in Figure 3(a). Adding nanorods to the mixture slightly shortens the first stage of the reaction (cf. Figure 2(b)) which is likely caused by the appearance of additional steric constraints in the mixture, but the fundamental character of the reaction does not change.

In the second stage of the reaction (usually, final products are obtained from epoxy materials only after several hours of curing or after postcuring at an elevated temperature), changes are no longer visible so well in the modulus representation, in any of the studied cases. A parameter found to be more sensitive in that phase is the real part of the electric permittivity at low frequencies. It does not display a relaxation profile that is so convenient for observation, but its value changes steadily during the entire observation time-reflecting the progress of the reaction-even though the rate of those changes varies with the curing progress. For further analysis, the normalized value $(\gamma)$ of the real part of the permittivity $(\varepsilon)$ was chosen:

$$
\gamma=\frac{\varepsilon-\varepsilon_{0}}{\varepsilon}
$$

where $\varepsilon$ is the current value and $\varepsilon_{0}$ is the initial value. This representation enables seeing the two-phase character of the reaction, both for curing with DDM and for curing with PA (Figure 3(b)). Curve fitting provides a tool to quantify and follow the rate changes of both the processes-not only in the initial phase but during the whole observation interval. It also enables comparing the curing parameters for mixtures with and without nanorods. However, the fundamental mechanisms of the curing reactions are different for PA and DDM and they require different fitting curves. Generally, a single exponential function appears in the description of first-order reactions. But higher-order reactions (including reactions in epoxy materials) are described by a somewhat more complex differential equation [34]:

$$
\frac{d \alpha}{d t}=k(T) \cdot f(\alpha)=k(T) \cdot \alpha^{m} \cdot(1-\alpha)^{n},
$$

where the conversion factor $(\alpha)$ depends on the reaction rate $k(T)$ and the exponents $n+m$ which determine the kinetic order. For curing with DDM, a relatively simple exponential model enables identification of two stages with clearly different reaction rates. The reaction process can be modelled by a double-decay function with two exponential terms describing the observed fast and slow phases of curing:

$$
\gamma=\gamma_{0}+A_{1} e^{-t / t_{1}}+A_{2} e^{-t / t_{2}},
$$


TABLE 2: Kinetic parameters of the reaction progress in various systems. Values determined from the best fit of the double-decay function (7) for mixtures with DDM and the Hill equation (8) for systems with PA.

\begin{tabular}{lccccc}
\hline Parameters & $\gamma_{0}$ & $A_{1}$ & $t_{1}$ & $A_{2}$ & $t_{2}$ \\
LCM/DDM & 0.545 & -0.138 & 2.6 & -0.407 & 334 \\
LCM/DDM_N & 0.217 & -0.057 & 9.0 & -0.160 & 66 \\
\hline Parameters & $P_{\mathrm{m}}$ & $K_{\mathrm{a}}$ & $K_{\mathrm{i}}$ & $H_{\mathrm{a}}$ & $H_{\mathrm{i}}$ \\
LCM/PA & 0.33 & 16 & 160 & 2.6 & 2.2 \\
LCM/PA_N & 0.48 & 13 & 250 & 1.8 & 0.7 \\
\hline
\end{tabular}

where $\gamma_{0}$ is offset, $A_{1}$ and $A_{2}$ are amplitudes, and $t_{1}$ and $t_{2}$ are time constants. The obtained fitting parameters are listed in Table 2. It is easy to notice that the parameter $A_{1}$ describing the first phase of the reaction is almost an order of magnitude smaller after addition of the nanofiller. Also, the characteristic times for the first stage of the reaction $\left(t_{1}\right)$ are much smaller than those for the second stage $\left(t_{2}\right)$, in both the mixtures.

Similar conclusions can be drawn from the observation of curing with pimelic acid as the curing agent, but the reaction model is quite different. The apparent two-stage reaction progress can be approximated in this case by biphasic Hill equation, used in biochemistry to describe the enzyme kinetics [35-37]:

$$
y=\frac{P_{\mathrm{m}}}{\left[1+\left(K_{\mathrm{a}} / x\right) H_{\mathrm{a}}\right] \cdot\left[1+\left(x / K_{\mathrm{i}}\right) H_{\mathrm{i}}\right]},
$$

where $P_{\mathrm{m}}$ is the maximum activity, $K_{\mathrm{a}}$ is the halfmaximal activity, $K_{\mathrm{i}}$ is the half-maximal inhibitory coefficient, $H_{\mathrm{a}}$ is the activation Hill coefficient, and $H_{\mathrm{i}}$ is the inhibitory Hill coefficient. To apply this formula to the experimental data from Figure 3(b), the generic variables $x$ and $y$ need to be replaced with the reaction time $t$ and the normalized permittivity $\gamma$, respectively. After adding nanorods, there is a visible growth of the $K_{\mathrm{i}}$ coefficient (connected with the factors inhibiting the reaction) which has an influence on deceleration of changes in the second phase of the reaction. The other parameters do not differ substantially. Thanks to the proposed representation one can easily follow not only the curing reaction kinetics but also the influence of the introduced modifiers on the reaction progress.

4.2. Properties of the Cured Resin and Its Composites. In the next part of the work, the dielectric properties of the products curing the investigated compound with DDM and PA are compared, including products modified by the addition of nanorods. Electrical and mechanical changes in the material at different temperatures can be described by analyzing the dielectric data represented in terms of the complex permittivity. Here, we chose to compare the properties of the studied composites by looking at the imaginary part of the permittivity, shown in a wide range of frequency and temperature in Figure 4.
The imaginary part of the permittivity is generally associated with dielectric losses in the material. The large growth of the imaginary part of the permittivity in both materials at low frequencies and high temperatures is due to the electric conductivity of the material (Figure 4). With decreasing temperature, the conductivity drops substantially and relaxation processes become visible. Along with further temperature drop, the $\alpha$-process vanishes and a broad $\beta$-process appears. In all the studied materials, the vanishing of the $\alpha$-process (which is related to the glass transition) is accompanied by a rapid change in the conductivity, which decreases by several orders of magnitude in the glassy phase.

The response of the cured material varies substantially for different curing agents, mirroring the differences already observed in the course of the curing process. The dielectric response of the pure epoxy matrix is generally similar to the response of the modified product; they only differ in detail, which will be analyzed below. In the mixtures of the epoxy resin with DDM, modified by adding the nanofiller, the $\alpha$-process is barely seen in the imaginary part of the dielectric permittivity at high temperatures because it is partially masked by high conductivity (Figure 4(a)). In the mixtures with nanorods, a growth of the imaginary part of the permittivity occurs at very low temperatures and high frequencies, next to the $\beta$ process - this hints towards a possible occurrence of another relaxation. However, the collected range of data is not sufficient for definitive confirmation of such an additional process in the materials with nanorods, or its closer analysis (Figure 4).

In the mixtures of the investigated resin with PA, both processes $(\alpha$ and $\beta$ ) are well visible; the $\alpha$-process is observed at temperatures lower than those of the products cured with DDM (Figure 4(b)). At low temperatures, a similar $\beta$-process appears in the mixtures both with and without the addition of nanorods.

As it was mentioned in Introduction, the $\alpha$-process is generally connected with the glass transition and enables estimation of the fragility index; the $\beta$-process reflects the mobility of molecular groups with large dipole moments and appears nearly always together with the $\alpha$-process. These relaxation processes can be analyzed more quantitatively by using the Havriliak-Negami (HN) formula with a conductivity term [38]:

$$
\varepsilon^{*}(\omega)-\varepsilon_{\infty}=-i \frac{\sigma_{0}}{\varepsilon_{0} \omega^{s}}+\sum_{k} \frac{\Delta \varepsilon_{k}}{\left(1+\left(i \omega \tau_{k}\right)^{\alpha_{k}}\right)^{\beta_{k}}}
$$

Here, $\varepsilon_{0}$ is the electric permittivity of vacuum, $\omega=2 \pi f$ is the radial frequency, and $\varepsilon_{\infty}$ is $\varepsilon^{\prime}$ at $f \rightarrow \infty$; the dielectric strength $\Delta \varepsilon$ is the difference between the high-frequency and low-frequency electric permittivities corresponding to the relaxation process under consideration, $\tau$ is the characteristic relaxation time, and $\alpha$ and $\beta$ are shape parameters of the relaxation time spectra; $\sigma_{0}$ denotes the DC conductivity and the parameter $s(s \leq 1)$ accounts for non-ohmic effects. The relaxation times for all the processes were 


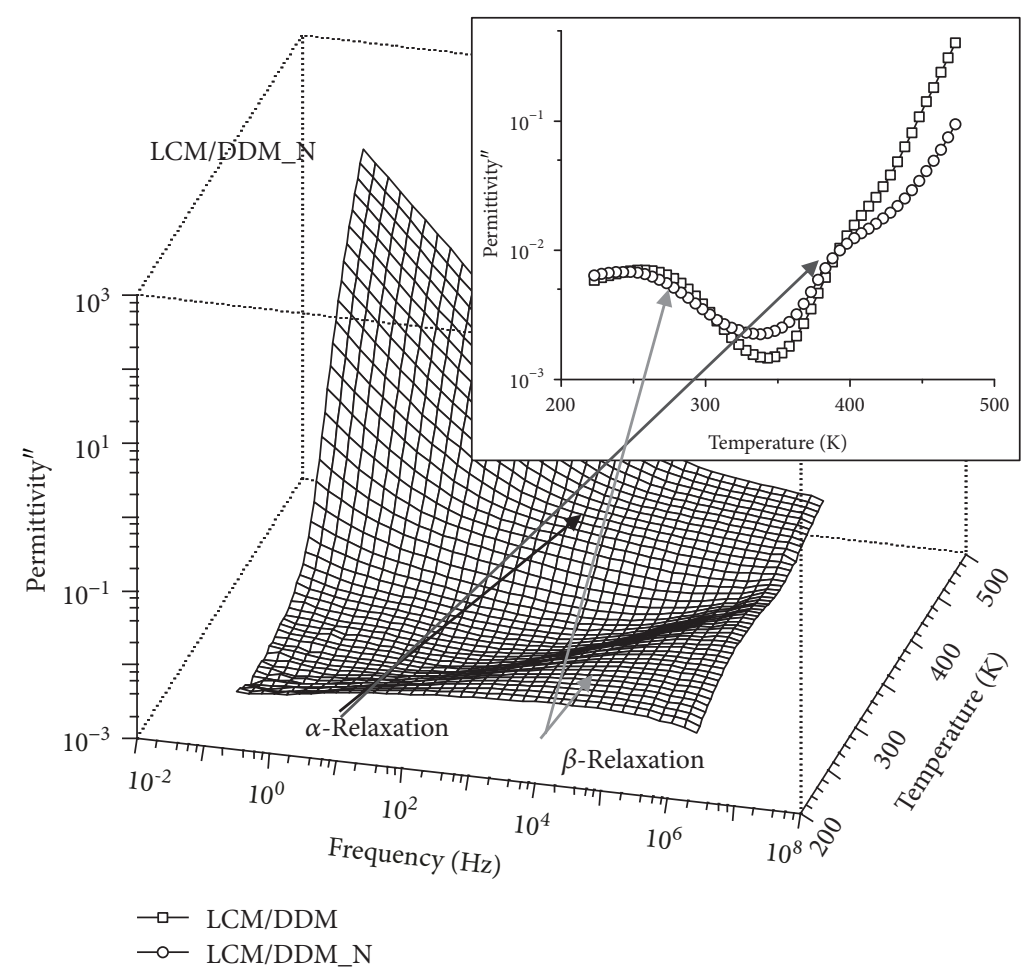

(a)

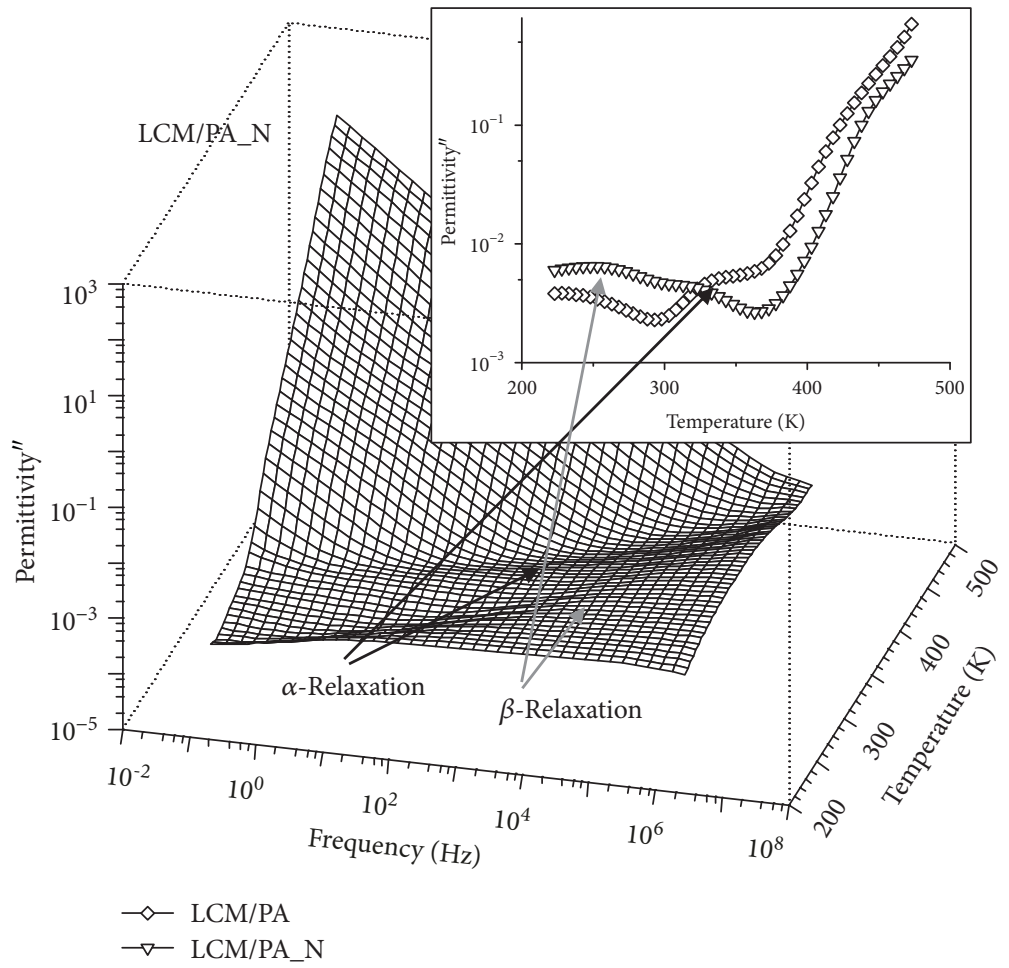

(b)

FIGURE 4: Imaginary part of the complex permittivity, at various temperatures: (a) LCM/DDM with nanorods; (b) LCM/PA with nanorods. Insets: comparative plots of $\varepsilon^{\prime \prime}(T)$ at $f=4 \mathrm{kHz}$ for mixtures cured with and without nanofillers.

determined by fitting the HN formula to experimental data, for each temperature at which these processes were visible. The use of the HN formula enables extraction of individual processes with their characteristic parameters. The relaxation times for all the mixtures, both cured with the acid and the amine, are shown in Figure 5. 


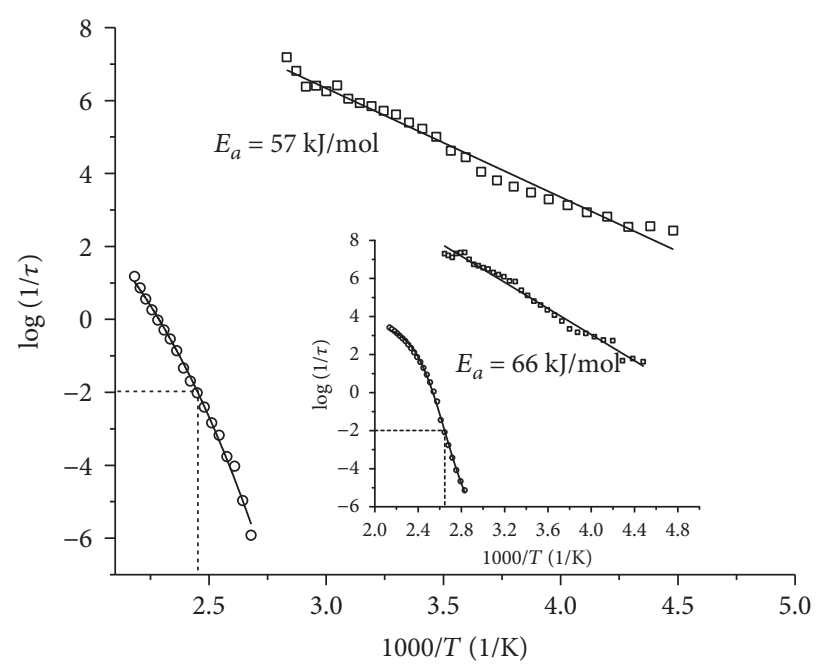

(a)

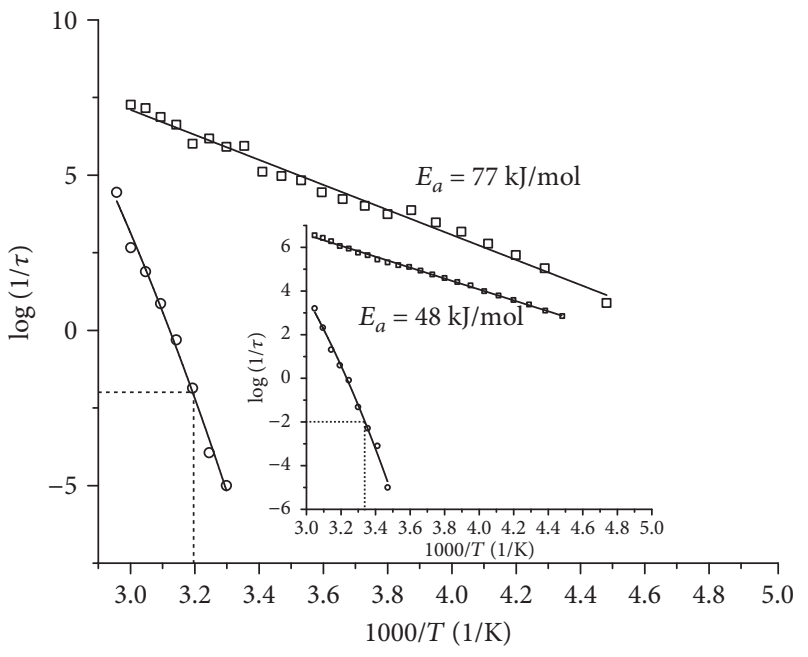

(b)

FIGURE 5: Temperature dependency of the relaxation times for all the observed processes, with the VFT formula fitted to the $\alpha$-processes and the Arrhenius formula fitted to the $\beta$-processes: (a) monomer cured with DDM and nanofiller additive (inset: pure LCM/DDM mixture); (b) monomer cured with PA and nanofiller additive (inset: pure LCM/PA mixture).

TABLE 3: Glass transition temperature $T_{\mathrm{g}}\left(\tau_{M}{ }^{\prime \prime}=10^{2} s\right)$ and fragility index $m$ for each of the investigated mixtures. Fragility index values obtained from VFT equation (11) and formula (13).

\begin{tabular}{lcccc}
\hline Parameters & $T_{\mathrm{g}}(\mathrm{K})$ & $D$ & $T_{0}(\mathrm{~K})$ & $m$ \\
\hline LCM/DDM & 378 & 2 & 337 & 76 \\
LCM/DDM_N & 408 & 12 & 275 & 35 \\
LCM/PA & 298 & 33 & 188 & 68 \\
LCM/PA_N & 313 & 48 & 194 & 90 \\
\hline
\end{tabular}

It is noteworthy that the $\beta$-process realizes the typical Arrhenius dependency [17-21]:

$$
\frac{1}{\tau}=\frac{1}{\tau_{0}} \cdot e^{-E_{a} / k T} .
$$

The $\alpha$-process, which deviates from that dependency, can be modelled by the VFT relation:

$$
\frac{1}{\tau}=\frac{1}{\tau_{0}} \cdot e^{-D T_{0} /\left(T-T_{0}\right)},
$$

where $D$ is a dimensionless quantity called the strength parameter and $T_{0}$ is the Vogel temperature [39].

The glass transition temperature $T_{\mathrm{g}}$ can be estimated from the vanishing of the $\alpha$-process. By convention, it is extracted from the VFT plot at the frequency of $10^{-2} \mathrm{~Hz}$; that approach was confirmed in many cases [17-21]. The parameters obtained from Figure 5 were collected in Table 3. The estimated $T_{\mathrm{g}}$ for the mixture of the monomer with DDM is $105^{\circ} \mathrm{C}$, whereas addition of the nanorods results in the growth of $T_{\mathrm{g}}$ to the value of $135^{\circ} \mathrm{C}$ (Figure 5(a)). Much lower temperatures were obtained for the mixtures cured with pimelic acid, which produced elastomers with $T_{\mathrm{g}}$ of $25^{\circ} \mathrm{C}$ for the plain mixture and $40^{\circ} \mathrm{C}$ for the mixture with the nanofiller (Figure 5(b)). The above conclusions are in good agreement with those obtained earlier from calorimetric data, though the values of $T_{\mathrm{g}}$ are not identical; this is linked with different cooling rates in the calorimetric studies and isothermal nature of dielectric measurements. Nevertheless, the addition of the nanofiller increased the vitrification temperature by $15^{\circ} \mathrm{C}$ in the materials cured with $\mathrm{PA}$ and by $30^{\circ} \mathrm{C}$ in the materials cured with DDM; this behavior was also noticed in the calorimetric study [31].

Another parameter describing the properties of the material is the fragility index, whose values typically lie in the range from about 16 for particularly hard substances to about 200 for brittle materials [23]. The fragility index is defined as $[23,39,40]$ :

$$
m=\left[\frac{d\left(\log _{10} \tau\right)}{d\left(T_{g} / T\right)}\right]_{T=T_{g}} .
$$

Knowing the parameters of the VFT equation, the fragility index can be calculated directly from the relation:

$$
m=\frac{D T_{0}}{2.3 \cdot T_{g}\left(1-T_{0} / T_{g}\right)^{2}} .
$$

The parameters calculated by fitting the VFT formula to the experimental data for all the mixtures are shown in Table 3. The apparent effect of adding nanorods is the increase of $T_{\mathrm{g}}$, regardless of the hardener used. On the contrary, the fragility index $m$ behaves differently for the two curing agents. It decreases after introduction of the nanofiller in the mixture cured with DDM; together with the increase of $T_{\mathrm{g}}$, this can be interpreted as the material is becoming harder. The value of $m$ increases after adding the nanorods in the 
more elastic mixtures, cured with PA; this suggests that the material becomes more brittle and less elastic.

The dielectric response of all the mixtures displays also a second relaxation, the $\beta$-process (Figure 4 ). The activation energy of that process changes after introduction of the nanofiller (Figure 5). The $\beta$-process is commonly associated with the motions of local dipolar groups. In the studied case, it is most likely linked with the dipolar carboxy groups in the monomer, because it appears in all the compositions, regardless of the curing agent. Although the activation energy of the observed process varies slightly among the studied mixtures, such variations may be due to differences in the energy barrier, reflecting differences in the internal structure of the network. A similar process was also observed in materials with different lengths of the mesogen [24]. An interesting case was found in the LCM/PA mixtures cured with the addition of nanorods, where an additional growth of the imaginary part of the permittivity was observed at high frequencies and low temperatures (Figure 4(b), inset). It may suggest that an additional process with very low activation energy, overlapping with the $\beta$-process, emerges in the samples containing the nanofiller. The possibility of such a process in the dielectric response can be explained by increased rigidity of the material after adding the nanofiller, shifting a fast relaxation process from high frequencies into the frequency range covered by the experiment. However, unequivocal interpretation of this growth of permittivity would require more detailed studies at low temperatures or at high frequencies.

\section{Conclusions}

The dynamics of the curing process observed in the dielectric spectroscopy was different in the materials cured with pimelic acid (PA) and 4,4'-diaminodiphenylmethane (DDM). The addition of the nanofiller did not change that dynamics but slightly slowed down the initial phase of the reaction.

Hard products were obtained from mixtures cured with DDM, whereas curing with PA yielded elastic materials. Dielectric observation of the $\alpha$-relaxation in the investigated epoxy matrix confirmed that the main factor determining the elasticity of the cured product is the choice of the hardener. The observation of the $\alpha$-relaxation in products with the addition of nanorods confirmed the increase of the vitrification temperature $T_{\mathrm{g}}$, regardless of the hardener used. The product obtained by adding nanorods to the mixture of the monomer with DDM was characterized by the highest rigidity.

The $\beta$-relaxation process was observed in all the mixtures. The suggested origin of the process is the motion of the dipolar carboxy groups in the mesogen.

\section{Data Availability}

The experimental data used to support the findings of this study are available from the corresponding author upon request.

\section{Conflicts of Interest}

The author declares that there is no conflict of interest regarding the publication of this paper.

\section{Acknowledgments}

The author is indebted to Beata Mossety-Leszczak, Krzysztof Lokaj, and Zbigniew Florjańczyk for providing the samples of liquid crystal monomer and the nanorods, and to Konrad Słaby for technical support. This research was performed as part of the employment of the author at Lodz University of Technology, supported by the Ministry of Science and Higher Education, Republic of Poland.

\section{References}

[1] E. P. Douglas, "Structure-property relationships in liquid crystalline thermosets," Journal of Macromolecular Science, Part C: Polymer Reviews, vol. 46, no. 2, pp. 127-141, 2006.

[2] A. Shiota and C. K. Ober, "Rigid rod and liquid crystalline thermosets," Progress in Polymer Science, vol. 22, no. 5, pp. 975-1000, 1997.

[3] T. S. Chung, Thermotropic Liquid Crystal Polymers: Thin-film Polymerization, Characterization, Blends, and Applications, Technomic Pub Co, Lancaster, PA, USA, 2001.

[4] A. Shalwan, M. Alajmi, and A. Alajmi, "Insulation characteristics of sisal fibre/epoxy composites," International Journal of Polymer Science, vol. 2017, Article ID 7312609, 6 pages, 2017.

[5] B. Mossety-Leszczak and M. Wlodarska, "Liquid-crystalline epoxy thermosets as matrices for ordered nanocomposites-a summary of experimental studies," Polymer Composites, vol. 38, no. 2, pp. 277-286, 2017.

[6] J. Liu, C. Wang, G. A. Campbell, J. D. Earls, and R. D. Priester, "Effects of liquid crystalline structure formation on the curing kinetics of an epoxy resin," Journal of Polymer Science Part A: Polymer Chemistry, vol. 35, no. 6, pp. 1105-1124, 1997.

[7] H. Körner, A. Shiota, T. J. Bunning, and C. K. Ober, "Orientation-on-demand thin films: curing of liquid crystalline networks in ac electric fields," Science, vol. 272, no. 5259, pp. 252-255, 1996.

[8] J. Y. Lee and J. Jang, "Anisotropically ordered liquid crystalline epoxy network on carbon fiber surface," Polymer Bulletin, vol. 59, no. 2, pp. 261-268, 2007.

[9] N. Suzuki, S. Kiba, and Y. Yamauchi, "Fabrication of epoxy composites with large-pore sized mesoporous silica and investigation of their thermal expansion," Journal of Nanoscience and Nanotechnology, vol. 12, no. 2, pp. 983-987, 2012.

[10] N. Suzuki, S. Kiba, and Y. Yamauchi, "Fabrication of mesoporous silica KIT-6/polymer composite and its low thermal expansion property," Materials Letters, vol. 65, no. 3, pp. 544-547, 2011.

[11] W. Yu, M. Qian, and H. Li, "Elastic and plastic properties of epoxy resin syntactic foams filled with hollow glass microspheres and glass fibers," Journal of Applied Polymer Science, vol. 133, no. 46, 2016.

[12] C. Carfagna, E. Amendola, and M. Giamberini, "Liquid crystalline epoxy based thermosetting polymers," Progress in Polymer Science, vol. 22, no. 8, pp. 1607-1647, 1997. 
[13] J. Y. Lee and J. Jang, "The effect of mesogenic length on the curing behavior and properties of liquid crystalline epoxy resins," Polymer, vol. 47, no. 9, pp. 3036-3042, 2006.

[14] P. Castell, A. Serra, M. Galià, M. Giamberini, and C. Carfagna, "Anisotropic thermosets from liquid-crystalline azomethynic epoxy resins and primary aromatic diamines," Journal of Polymer Science Part A: Polymer Chemistry, vol. 41, no. 1, pp. 1-12, 2003.

[15] D. Prevosto, S. Capaccioli, S. Sharifi, K. Kessairi, M. Lucchesi, and P. A. Rolla, "Secondary dynamics in glass formers: relation with the structural dynamics and the glass transition," Journal of Non-Crystalline Solids, vol. 353, no. 47-51, pp. 4278-4282, 2007.

[16] K. Mohomed, T. G. Gerasimov, F. Moussy, and J. P. Harmon, "A broad spectrum analysis of the dielectric properties of poly(2-hydroxyethyl methacrylate)," Polymer, vol. 46, no. 11, pp. 3847-3855, 2005.

[17] J. Rault, "Relaxation of glasses: the Kohlrausch exponent," Journal of Non-Crystalline Solids, vol. 357, no. 2, pp. 339345, 2011.

[18] J. Rault, “Origin of the Vogel-Fulcher-Tammann law in glassforming materials: the $\alpha-\beta$ bifurcation," Journal of NonCrystalline Solids, vol. 271, no. 3, pp. 177-217, 2000.

[19] J. Rault, "Remarks on the Kohlrausch exponent and the Vogel-Fulcher-Tamann law in glass-forming materials," Journal of Non-Crystalline Solids, vol. 260, no. 1-2, pp. 164-166, 1999.

[20] F. Kremer and A. Schönhals, Broadband Dielectric Spectroscopy, Springer, Berlin, Germany, 2003.

[21] G. P. Johari and M. Goldstein, "Viscous liquids and the glass transition. II. Secondary relaxations in glasses of rigid molecules," The Journal of Chemical Physics, vol. 53, no. 6, pp. 2372-2388, 1970.

[22] P. B. Macedo, C. T. Moynihan, and R. Bose, "The role of ionic diffusion in polarization in vitreous ionic conductors," Physics and Chemistry of Glasses, vol. 13, pp. 171-179, 1972.

[23] B. Gonzalo, J. L. Vilas, M. San Sebastián et al., "Electric modulus and polarization studies on piezoelectric polyimides," Journal of Applied Polymer Science, vol. 125, no. 1, pp. 67-76, 2012.

[24] M. Wlodarska, A. Maj, B. Mossety-Leszczak et al., "Liquid crystal epoxy resins based on biphenyl group cured with aromatic amines - studied by dielectric spectroscopy," Journal of Polymer Research, vol. 20, no. 9, 2013.

[25] S. D. Senturia and N. F. Sheppard, "Dielectric analysis of thermoset cure," in Epoxy Resins and Composites IV, vol. 80 of Advances in Polymer Science, pp. 1-47, 1986.

[26] D. Kranbuehl, S. Delos, E. Yi et al., "Dynamic dielectric analysis: nondestructive material evaluation and cure cycle monitoring," Polymer Engineering and Science, vol. 26, no. 5, pp. 338-345, 1986.

[27] W. H. Jeu, Physical Properties of Liquid Crystalline Materials, Gordon and Breach, London, UK, 1980.

[28] A. Kozak, J. K. Mościcki, and G. Williams, "On dielectric relaxation in liquid crystals," Molecular Crystals and Liquid Crystals, vol. 201, no. 1, pp. 1-12, 1991.

[29] H. Kresse, "Dielectric behavior of liquid crystals," Advanced Liquid Crystal, vol. 6, p. 109, 1983.

[30] J. Jadżyn, G. Czechowski, R. Douali, and C. Legrand, "Molecular model of the dielectric relaxation in nematic liquid crystals," in Proceedings Volume 4147, Liquid Crystals:
Chemistry, Physics, and Applications, pp. 176-183, Krynica Zdroj, Poland, 2000.

[31] B. Mossety-Leszczak, H. Galina, M. Włodarska, M. Kowalik, K. Łokaj, and Z. Florjańczyk, "Żywice i sieci epoksydowe o właściwościach anizotropowych,” Polimery, vol. 54, no. 639, pp. 719-726, 2009.

[32] Z. Florjańczyk, A. Wolak, M. Dębowski et al., "Organically modified aluminophosphates: transformation of boehmite into nanoparticles and fibers containing aluminodiethylphosphate tectons," Chemistry of Materials, vol. 19, no. 23, pp. 5584-5592, 2007.

[33] Z. Florjańczyk, A. Lasota, A. Wolak, and J. Zachara, "Organically modified aluminum phosphates: synthesis and characterization of model compounds containing diphenyl phosphate ligands," Chemistry of Materials, vol. 18, no. 7, pp. 19952003, 2006.

[34] E. Amendola, C. Carfagna, and M. Giamberini, "Role of curing agent on the nature of the mesophase and the properties of mesogenic epoxy resins," in Liquid Crystalline Polymer Systems: Technological Advances, A. I. Isayev, T. Kyu, and S. Z. D. Cheng, Eds., American Chemical Society, Washington, DC, USA, 1996.

[35] J. M. Hutzler and T. S. Tracy, "Atypical kinetic profiles in drug metabolism reactions," Drug Metabolism and Disposition, vol. 30, no. 4, pp. 355-362, 2002.

[36] B. Wu, R. Ako, and M. Hu, "A useful Microsoft Excel add-in program for modeling steady-state enzyme kinetics," Pharmaceutica Analytica Acta, vol. 1, no. S1, 2011.

[37] K. E. Kenworthy, S. E. Clarke, J. Andrews, and J. B. Houston, "Multisite kinetic models for CYP3A4: simultaneous activation and inhibition of diazepam and testosterone metabolism," Drug Metabolism and Disposition, vol. 29, no. 12, pp. 16441651, 2001.

[38] S. Havriliak and S. Negami, “A complex plane representation of dielectric and mechanical relaxation processes in some polymers," Polymer, vol. 8, pp. 161-210, 1967.

[39] R. Böhmer, K. L. Ngai, C. A. Angell, and D. J. Plazek, "Nonexponential relaxations in strong and fragile glass formers," The Journal of Chemical Physics, vol. 99, no. 5, pp. 4201-4209, 1993.

[40] R. Böhmer and C. A. Angell, "Correlations of the nonexponentiality and state dependence of mechanical relaxations with bond connectivity in Ge-As-Se supercooled liquids," Physical Review B, vol. 45, no. 17, pp. 10091-10094, 1992. 


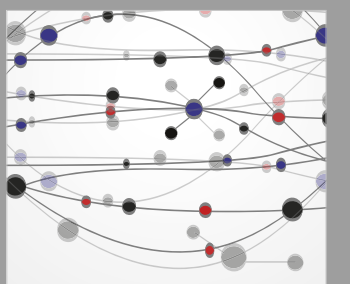

The Scientific World Journal
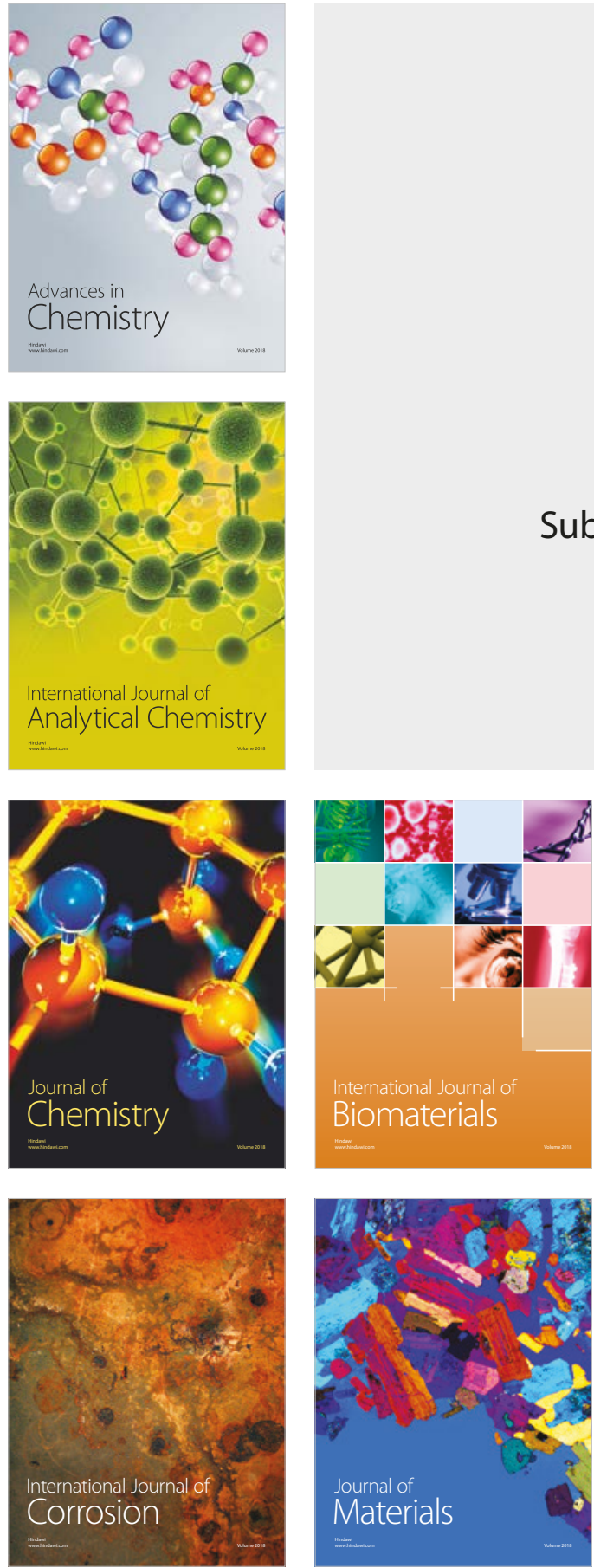

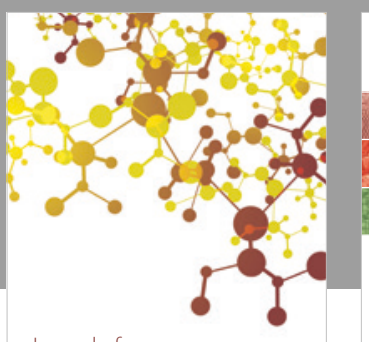

Journal of

Applied Chemistry
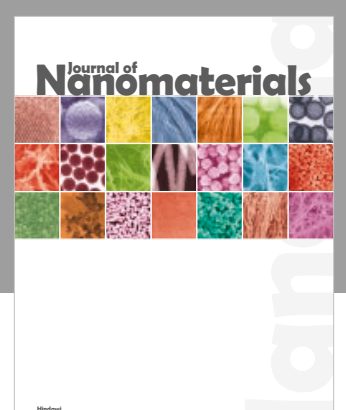

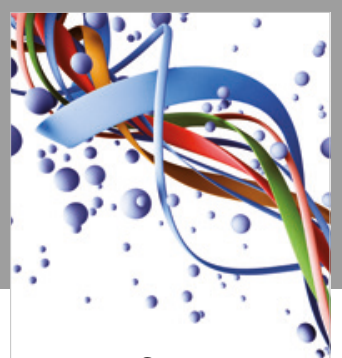

Scientifica

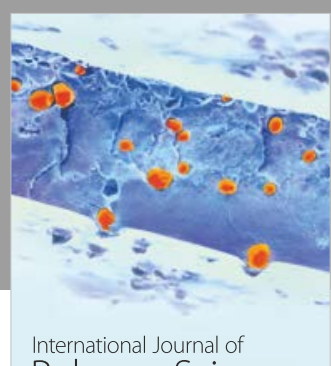

Polymer Science

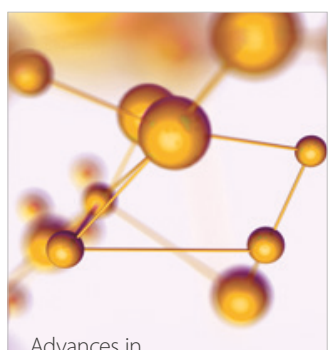

Physical Chemistry
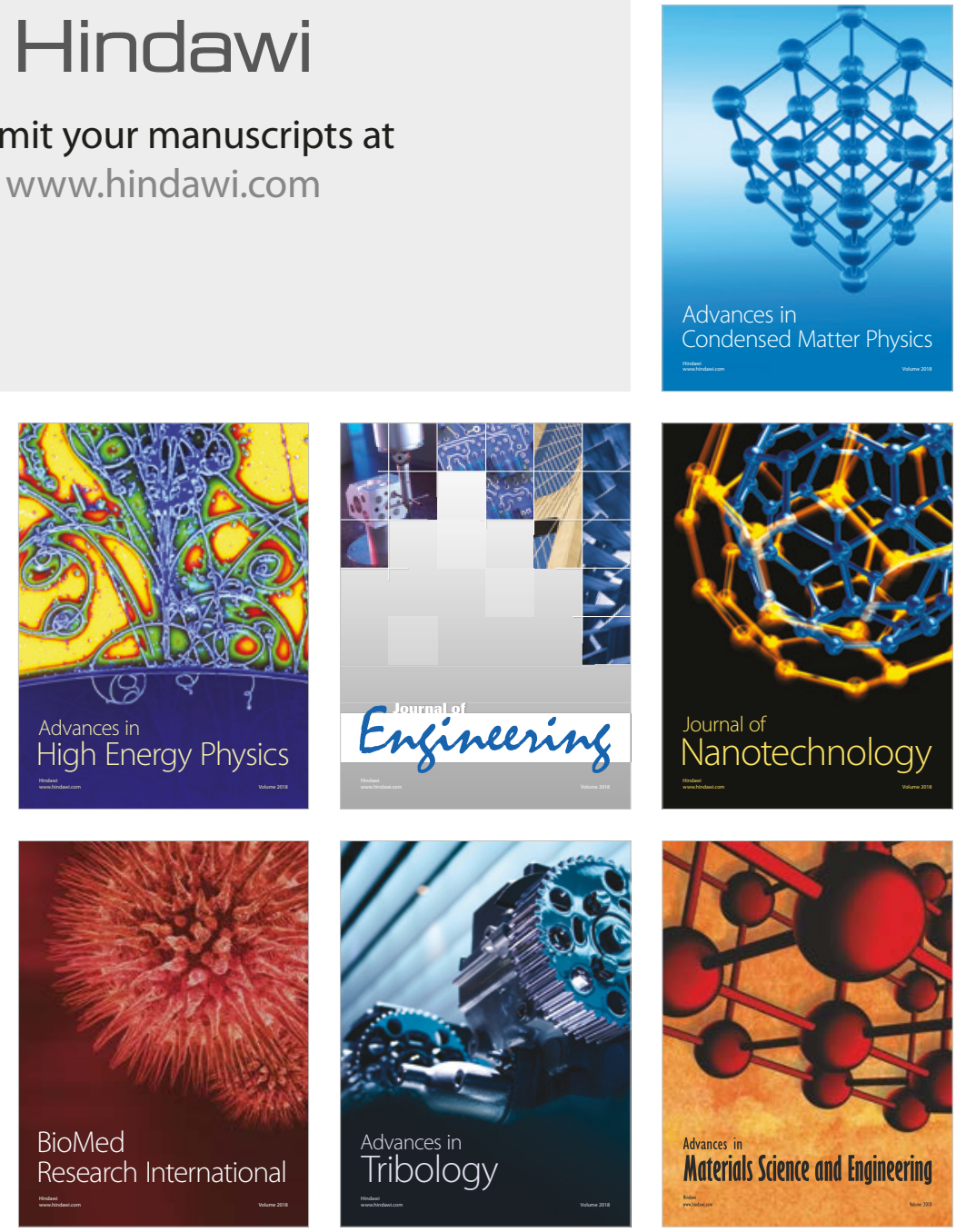